\title{
Investigating bacterial endosymbiosis in Leptogorgia virgulata using a molecular and SEM approach
}

\author{
Sarah Abboud, * Roni Kingsley, ** Lori Spicer, ***and Carolyn Marks **** \\ * Graduate student at Northeastern University, Nahant, MA, 01908 \\ ** Department of Biology, University of Richmond, Richmond, VA 23173 \\ *** Department of Biology, University of Richmond, Richmond, VA 23173 \\ **** Department of Biological Imaging, University of Richmond, VA 23173
}

The common sea-whip, Leptogorgia virgulata is reported to be asymbiotic in terms of endosymbiotic dinoflagellates. Though we tend to believe that L. virgulata uses possible bacterial symbionts as a mechanism to reduce acidic environments within the gorgonian tissue to form calcium carbonate spicules. This study investigates urease production by bacteria within $L$. virgulata to reduce acidity. The goal of the study is to characterize possible bacterial symbionts and using SEM determine locality within $L$. virgulata tissue. Possible microbe symbionts were isolated from $L$. virgulata tissue through a series of dilutions where bacteria colonies unique to surface bacteria were cultured and tested for urease production. Bacteria that were positive for urease production were then prepared for SEM analysis and a series of magnification SEM references for cultured bacteria were compared to freeze fractured L. virgulata SEM prepared samples to determine possible microbe symbiont location in the gorgonian tissue. Possible bacterial endosymbionts were characterized using univ518r primers typical for identifying universal bacteria. Preliminary molecular data suggests there are unique microbes inhabiting L. virgulata tissue, though their location is still being investigated. 